TPI-MINN-95-08/T

NUC-MINN-95-11/T

HEP-UMN-TH-1337

April 1995

\title{
Really Computing Non-perturbative Real Time Correlation Functions
}

\author{
Dietrich Bödeker and Larry McLerran \\ School of Physics and Astronomy, University of Minnesota, Minneapolis, MN 55455, USA \\ and \\ Andrei Smilga \\ ITEP, B.Cheremushkinskaya 25, Moscow 117259, Russia
}

\begin{abstract}
It has been argued by Grigoriev and Rubakov that one can simulate real time processes involving baryon number non-conservation at high temperature using real time evolution of classical equations, and summing over initial conditions with a classical thermal weight. It is known that such a naive algorithm is plagued by ultraviolet divergences. In quantum theory the divergences are regularized, but the corresponding graphs involve the contributions from the hard momentum region and also the new scale $\sim g T$ comes into play. We propose a modified algorithm which involves solving the classical equations of motion for the effective hard thermal loop Hamiltonian with an ultraviolet cutoff $\mu \gg g T$ and integrating over initial conditions with a proper thermal weight. Such an algorithm should provide a determination of the infrared behavior of real time correlation function $<Q(t) Q(0)>_{T}$ determining the baryon violation rate. Hopefully, the results obtained in this modified algorithm would be cutoff-independent.
\end{abstract}




\section{Introduction}

Sphaleron processes in electroweak theory mediate baryon number violating processes, and have been computed to be significant at high temperatures [1] - [6] This computation involves estimating the probability of a classical thermal fluctuation evolving over the top of an energy barrier which separates topologically distinct phases of electroweak theory. At very high temperatures, where the masses of the $\mathrm{W}$ and $\mathrm{Z}$ boson can be ignored, the field configurations which dominate the contribution have a typical size of order $1 / \alpha_{W} T$ [6]. This is the length scale on which non-perturbative physics is important, and there is no known way of doing a weak coupling estimate of the effect.

In a provocative paper by Grigoriev and Rubakov [7], a numerical algorithm was suggested for the estimation of the baryon number violation rate. They argue that at high temperature, the dominant process for sphaleron processes involves transitions of fields over the energy barrier. The dominant transitions should therefore be classically allowed thermal processes. Since the field configurations useful for sphaleron processes are long wavelength and involve many quanta, it is reasonable to assume that the evolution of the field configuration over the top of the barrier is in fact classical.

If the evolution of the fields is classical, then they will be described by classical equations of motion with given initial conditions. These initial conditions are determined by a classical thermal ensemble where the initial distribution of fields and their conjugate momenta are computed in a Monte-Carlo simulation.

This method was used to compute the rate of sphaleron transitions in electroweak theory at temperature higher than the electroweak phase transition temperature [8]. It was also tested in two dimensional models [9] - [10]. Although the two dimensional results looked convincing, there have remained a number of plaguing problems associated with obtaining correct dependences on effective temperature dependent masses, and these masses depend on the ultraviolet region of the theory where, as we will see, there are problems associated with treating the theory classically. The $3+1$ dimensional simulations of electroweak theory are plagued by ultraviolet singularities associated with making the spatial lattice spacing smaller, and one may worry whether any quantitative numerical conclusions are warranted.

It is easy to understand why there are problems associated with taking the zero lattice spacing limit in such simulations. In the second section, we shall present a detailed mathematical argument in the context of $\phi^{4}$ theory . In the remainder of the Introduction, we shall present heuristic arguments which display the nature of the problem.

It has been well known since Rayleigh, Jeans, and Planck that, if one tries to describe a bath of photons classically, the free energy is ultraviolet divergent and depends on the cutoff. To see this, consider the energy density for a gas of scalar 
massless bosons. This density at high temperatures behaves as

$$
E=\int \frac{d^{3} p}{(2 \pi)^{3}} \frac{|\mathbf{p}|}{e^{\beta|\mathbf{p}|}-1}=\frac{\pi^{2} T^{4}}{30}
$$

On the other hand, if we try to compute the thermodynamic characteristics of the system classically, we have the functional integral representation

$$
Z^{c l}=\int[d \phi][d \Pi] \exp \{-\beta H\}
$$

where the Hamiltonian is

$$
H=\int d^{3} x\left\{\frac{1}{2} \Pi(x)^{2}+\frac{1}{2}(\nabla \phi(x))^{2}\right\}
$$

The results are ultraviolet divergent. For the energy density we get

$$
E^{c l}(T)=\frac{T}{6 \pi^{2}} \mu^{3}
$$

where $\mu$ is a momentum ultraviolet cutoff (The simplest way to get this result is to substitute the Bose distribution function in Eq.(1.1) by its classical limit $T /|\mathbf{p}|$ ). This is the well known classical Rayleigh-Jeans divergence and was one of the original motivations for quantum mechanics.

Ultraviolet divergences also crop up in other quantities. Consider e.g. the correlator $<\phi(x, t) \phi(0)>_{T}$ in $\phi^{4}$ theory.

This bears on what we discuss here since the quantity of interest defining baryon nonconservation rate in the standard model is a similar ( but, of course, more complicated) correlator

$$
C(t)=<[Q(t)-Q(0)] Q(0)>_{T}
$$

where

$$
Q(t)=\frac{g^{2}}{32 \pi^{2}} \epsilon_{i j k} \int d^{3} x\left(F_{i j}^{a} A_{k}^{a}-\frac{g}{3} \epsilon^{a b c} A_{i}^{a} A_{j}^{b} A_{k}^{c}\right)
$$

is the Chern-Simons winding number of the $S U(2)$ gauge field configuration. The expectation is that the correlator (1.5) behaves as $C(t) \sim \Gamma V|t|$ at large $|t| \gg$ $\left(\alpha_{W} T\right)^{-1}$ where $\Gamma$ is the baryon number nonconservation rate per unit volume and is estimated as [6]

$$
\Gamma^{\Delta B \neq 0}(T)=\kappa\left(\alpha_{W} T\right)^{4}
$$

We take the interaction term in the action as

$$
S=\int d t \int d^{3} x \frac{\lambda}{4 !} \phi^{4}(x)
$$


This yields in lowest order, the diagram shown in Figure 1.

In this paper, all perturbative calculations are performed consistently in the real time formalism which is the most suitable tool to study kinetic properties in thermal medium. In its complete form the formalism has been developed in 11, 12]. It involves some intricacies, one should carefully distinguish the retarded, the advanced and the so called $\mathrm{P}$-component of the Green's function $\mathrm{H}$. But we do not really need to go into details here because, for our purposes (we will be interested only with 1-loop graphs and only with the real part thereof), it almost suffices to use the simplified version of the formalism due to Dolan and Jackiw [17] (what almost means is explained in the Appendix A). In Dolan-Jackiw formalism the thermal propagators present the sum of two terms: the standard zero-temperature propagator and the temperature-dependent term which reflects the presence of real particles in the heat bath (alias, the temperature insertion). In particular, the thermal propagator of massless scalar is

$$
D_{T}(p)=\frac{i}{p^{2}+i 0}+2 \pi \delta\left(p^{2}\right) \frac{1}{e^{\beta\left|p_{0}\right|}-1}
$$

The temperature-dependent contribution in the polarization operator in Fig. [ is

$$
\frac{\lambda}{2} \int \frac{d^{3} p}{(2 \pi)^{3}|\mathbf{p}|\left(e^{\beta|\mathbf{p}|}-1\right)}=\frac{\lambda T^{2}}{24}
$$

which is the familiar temperature renormalization of the scalar mass. (Here and in the following, we ignore the infinite ultraviolet mass renormalization in the zerotemperature theory which we assume to be cancelled by a proper counterterm.) Let us try to calculate the same graph in classical theory. It is ultraviolet divergent $\$$.

$$
M_{c l}^{2}(T)=\frac{\lambda T}{2} \int \frac{d^{3} p}{(2 \pi)^{3}|\mathbf{p}|^{2}}=\lambda \frac{T \mu}{4 \pi^{2}}
$$

Note that the method of [7]- [10] where the correlator (1.5) was estimated by solving the classical equations of motion with the given initial conditions which then were averaged over by integrating over classical phase space with the weight $\exp \left\{-\beta H^{c l}\right\}$

${ }^{1}$ The review can be found in [13, 14]. For applications of the formalism to hot gauge theories see e.g. [15, 16] .

${ }^{2} \mathrm{Up}$ to now, we assumed that the tree-level mass of the scalar field is zero, but the result (1.11) holds also for the theory with nonzero mass $M_{0}$ if $\mu \gg M_{0}$. For the one-loop estimate (1.11) to be stable with respect to higher-order corrections, the characteristic momenta in the integral $|\mathbf{p}| \sim \mu$ should also be much larger than the full quantum temperature-induced mass $(1.10): \mu \gg \lambda^{1 / 2} T$. 
like in (1.2) is equivalent in perturbation theory to calculating the classical graphs. In Sect.2, we shall illustrate this explicitly for $\lambda \phi^{4}$ theory.

The way out of this difficulty suggested in [8] was to choose $\mu \sim T$. The particular proportionality coefficient was fixed by the requirement that the classical and quantum expressions for the energy density (the analogs of (1.1) and (1.4)) coincide. Note, however, that choosing $\mu$ in such a way, we would still have a mismatch between the classical (1.11) and quantum (1.10) expressions for the mass renormalization. This mismatch is a manifestation of a simple fact that the classical description is justified only for the low momentum modes. But, as we have seen, the low-energy sector of the theory does not entirely decouple from the high momentum modes. They get entangled together. Thus, the procedure used in 8 may be not reliable p $^{2}$.

Our main observation, however, is that low momentum modes and high momentum modes can in principle be disentangled. The correct procedure should be the following. Let us introduce an intermediate scale $\mu$ such that $g T \ll \mu \ll T$ and treat the modes with momenta $|\mathbf{p}|>\mu$ and $|\mathbf{p}|<\mu$ in a different way. The high momentum modes can be explicitly integrated over leading to the effective hard thermal loop Hamiltonian for the low momentum modes [18]-[20]. As the low energy bound for momenta circulating in the loop is not zero but is equal to $\mu$, this effective Hamiltonian would involve also some counterterms depending on the parameter $\mu$. With the effective Hamiltonian in hand, one can return back to the original GR procedure, solve the effective equations of motion, and average over initial conditions with the weight $\exp \left\{-\beta H^{e f f}\right.$ (phase space) $\}$. The ultraviolet 3-dim momentum cutoff should be chosen to be of order of $\mu$. The final result will be $\mu$-independent 国.

We emphasize that the success of this modified procedure depends on two circumstances:

1. For high momentum modes, the classical treatment is wrong, but these modes can be taken into account perturbatively.

\footnotetext{
${ }^{3}$ This question is, however, not clear at present. We return to the discussion of this issue in the last section. We want also to emphasize that the violent power ultraviolet divergences in $m^{c l}(T)$ and other parameters of the effective hight- $T$ Hamiltonian are spesific for 4-dimensional theories. In two-dimensional case considered in the first Grigoriev and Rubakov paper, the situation is much more benign.

${ }^{4}$ We were able to construct such a $\mu$-independent algorithm explicitly only for the scalar theory. In Ref. [21] such an algorithm has been discussed for a scalar theory in $(2+1)$ dimensions. The implementation of this idea in the gauge theory meets serious technical difficulties. We shall discuss it at length later.
} 
2. For low momentum modes, perturbation theory breaks down and the classical real time equations of motion are essentially non-perturbative. They cannot be evaluated by weak coupling methods. But the classical approach is justified here, and one can do a numerical calculation in the GR spirit.

Note that the method of separating hard and soft modes where the former can be treated perturbatively while the latter cannot, but the influence of hard modes on the soft mode physics is taken into account, is a standard one in many physical problems. E.g., it is the basis of the operator product expansion technique and sum rules in QCD [22].

In this paper, we first restrict ourselves with an illustrative analysis for $\lambda \phi^{4}$ theory. Later we outline the procedure to be used for gauge theories. Note that the situation is much more complicated in gauge theories when one computes the real time evolution of classical fields. For computing the real time evolution of the gauge fields, one obtains Feynman diagrams of the type shown in Fig. 2. When these diagrams are computed, one obtains a correction of the form

$$
\alpha T^{2} F^{\mu \nu}\left(k^{0} /|\mathbf{k}|\right)
$$

where $k^{\mu}$ is the external momentum. This function has a nontrivial dependence on $k^{0} /|\mathbf{k}|$. For $|\mathbf{k}| \sim g T$, this contribution is as large as the free particle kinetic energy terms, $g^{\mu \nu} k^{2}-k^{\mu} k^{\nu}$. One can show, however, that so long as $k^{0} \geq g T$ and $|\mathbf{k}| \geq g T$ the contribution of higher-order loops in the two-point function and other irreducible vertices is still suppressed and perturbation theory works. The shorthand for all these vertices is called the Braaten-Pisarski effective Lagrangian [18].

The main problem which distinguishes hot gauge theories compared to the simple $\lambda \phi^{4}$ case is that this effective Lagrangian is highly nonlocal. One cannot straightforwardly write the effective theory in a Hamiltonian form and write differential equations of motion for real time evolution of initial thermal gauge field configurations. However, in the recent works [19, 20, 23] this theory has been written in the local Hamiltonian form. The price which one had to pay is the introduction of additional variables the integration over which restores the original nonlocalities. Still, the local Hamiltonian description of hot gauge theories exists. Unfortunately, such a local effective Hamiltonian exists only if performing the integration in the hard thermal loops over all loop momenta (without infrared cutoff $\mu$ ). As was already mentioned, we were not able to derive $\mu$-dependent piece in the effective HTL Hamiltonian. Our hope is, however, that the contribution of these unknown terms in the baryon nonconservation rate in interest is suppressed.

The organization of this paper is as follows: In the second section, we reconsider the formalism of Grigoriev and Rubakov, and show explicitly how the ultraviolet cutoff dependence arises. In the Section 3 we use this formalism to compute the 
lowest order contribution to the propagator in the $\lambda \phi^{4}$ theory. In the fourth section we present a path integral formalism for computing real time correlation functions by a method which in lowest order approximation gives a result similar to that of Grigoriev and Rubakov. The effects due to hard modes are discussed in Sect.4. We argue that, for most practical purposes, one can evaluate the contribution of high momentum modes in a Gaussian approximation to the functional integral representation for the real time correlation function. We explicitly demonstrate this for $\lambda \phi^{4}$ theory. We show that this results in a formalism similar to that of Grigoriev and Rubakov except that one should use the effective hard thermal loop Lagrangian plus some counterterms needed to render the three dimensional classical equations finite. In Sect.5, we discuss hot gauge theories and, in particular, the kinetic equation approach and the Nair's effective Hamiltonian which may be the basis for the correct analysis of the problem of interest. We also discuss the kinematic limits in which our computation is valid. In the last section, we summarize our results. We describe what will be needed to be done before realistic computations for $3+1$ dimensional gauge theories may be performed.

There are also two technical Appendices devoted to two unsuccessful attempts to isolate $\mu$-dependent terms in the effective Hamiltonian for hot Yang-Mills theory. In Appendix A we isolate the leading cutoff-dependent terms in the soft multigluon thermal vertices with momentum infrared cutoff. Unfortunately, such a cutoff breaks gauge invariance. As a result, the $\mu$-dependent terms in the effective lagrangian are not gauge invariant, and the classical problem cannot be consistently posed. In Appendix B we display the problems one meets when trying to do the same with a lattice cutoff (here the problems are of the opposite kind. The lattice regularization is gauge-invariant, but to isolate the $\mu$-dependent pieces is very difficult technically) and perform some illustrative calcultations for hot scalar QED.

\section{Ultraviolet Problems}

In this section, we elucidate why the procedure of Ambjorn et al. doesn't properly take into account the high momentum modes with $k \sim T$. This is demonstrated for the case of a scalar theory with the action

$$
S=\int d^{4} x\left\{\frac{1}{2}\left(\partial_{\mu} \phi(x)\right)^{2}-\frac{1}{2} M_{0}^{2} \phi^{2}(x)-\frac{\lambda}{4 !} \phi^{4}(x)\right\}
$$

The object we want to compute is the real time correlation function

$$
C(t, \mathbf{k}, \mathbf{p})=<\phi(t, \mathbf{k}) \phi(0, \mathbf{p})>_{T}
$$

where

$$
\phi(t, \mathbf{k})=\int d^{3} x e^{-i \mathbf{k x}} \phi(t, \mathbf{x})
$$


In general, one can consider correlation functions of composite operators, but for the points we wish to make, it is sufficient to consider correlation functions of the elementary boson field.

The procedure of Grigoriev and Rubakov is equivalent to the following: Assume an initial condition which has been determined by random selection from a classical thermal distribution, that is, with weight

$$
\exp \left\{-\beta H\left(\Pi^{\text {in }}, \phi^{\text {in }}\right)\right\}
$$

where $\Pi$ is the momentum conjugate to $\phi$. If we know $\Pi^{\text {in }}$ and $\phi^{\text {in }}$, we have the initial conditions needed for solving the classical equations of motion.

The next part of the recipe is to solve the classical equations of motion. This then determines $\Pi(t, \mathbf{k})$ and $\phi(t, \mathbf{k})$. To compute the correlation function, one then has to average over the initial conditions.

The equation of motion

$$
\begin{aligned}
& \left(\frac{d^{2}}{d t^{2}}+\mathbf{k}^{2}+M_{0}^{2}\right) \phi(t, \mathbf{k}) \\
& =-\frac{\lambda}{3 !} \int \frac{d^{3} k_{1}}{(2 \pi)^{3}} \frac{d^{3} k_{2}}{(2 \pi)^{3}} \phi\left(t, \mathbf{k}_{1}\right) \phi\left(t, \mathbf{k}_{2}\right) \phi\left(t, \mathbf{k}-\mathbf{k}_{1}-\mathbf{k}_{2}\right)
\end{aligned}
$$

is solved perturbatively:

$$
\phi(t, \mathbf{k})=\phi_{1}(t, \mathbf{k})+\phi_{2}(t, \mathbf{k})+\cdots
$$

$\phi_{1}(t, \mathbf{k})$ is a superposition of plane waves:

$$
\phi_{1}(t, \mathbf{k})=\frac{1}{2 \omega_{k}}\left(a(\mathbf{k}) e^{-i\left(\omega_{k} t-\mathbf{k x}\right)}+a^{*}(-\mathbf{k}) e^{i\left(\omega_{k} t-\mathbf{k x}\right)}\right)
$$

where $a, a^{*}$ are determined by the initial conditions and

$$
\omega_{k}=\sqrt{\mathbf{k}^{2}+M_{\mathrm{cl}}^{2}}
$$

The mass $M_{\mathrm{cl}}$ is related to the tree level mass by

$$
M_{\mathrm{cl}}^{2}=M_{0}^{2}+\delta M_{\mathrm{cl}}^{2}
$$

The mass counterterm $\delta M_{\mathrm{cl}}^{2}$ will be determined below. $\phi_{2}(t, \mathbf{k})$ is obtained by solving

$$
\begin{array}{r}
\left(\frac{d^{2}}{d t^{2}}+\mathbf{k}^{2}+M_{\mathrm{cl}}^{2}\right) \phi_{2}(t, \mathbf{k})=-\frac{\lambda}{3 !} \int \frac{d^{3} k_{1}}{(2 \pi)^{3}} \frac{d^{3} k_{2}}{(2 \pi)^{3}} \phi_{1}\left(t, \mathbf{k}_{1}\right) \phi_{1}\left(t, \mathbf{k}_{2}\right) \\
\phi_{1}\left(t, \mathbf{k}-\mathbf{k}_{1}-\mathbf{k}_{2}\right)+\delta M_{\mathrm{cl}}^{2} \phi_{1}(t, \mathbf{k})
\end{array}
$$


with the initial conditions

$$
\phi_{2}(0, \mathbf{k})=0, \quad \frac{d}{d t} \phi_{2}(0, \mathbf{k})=0
$$

To evaluate the Green's function (2.2), we have to average $\phi^{\text {in }}(\mathbf{p})\left[\phi_{1}(t, \mathbf{k})+\phi_{2}(t, \mathbf{k})\right]$ over the initial condition with the weight (2.4):

$$
\begin{aligned}
C_{\mathrm{cl}}(t, \mathbf{k}, \mathbf{p})= & Z_{\mathrm{cl}}^{-1} \int\left[d \Pi^{\mathrm{in}}\right]\left[d \phi^{\mathrm{in}}\right] \exp \left\{-\beta H_{0}\left[\Pi^{\mathrm{in}}, \phi^{\mathrm{in}}\right]\right\} \\
& \left(1+\int d^{3} x\left(\frac{\lambda}{4 !}\left(\phi^{\mathrm{in}}\right)^{4}(\mathbf{x})-\frac{1}{2} \delta M_{\mathrm{cl}}^{2}\left(\phi^{\mathrm{in}}\right)^{2}(\mathbf{x})\right)\right) \\
& \phi^{\mathrm{in}}(\mathbf{p})\left(\phi_{1}(t, \mathbf{k})+\phi_{2}(t, \mathbf{k})\right)
\end{aligned}
$$

where

$$
H_{0}[\Pi, \phi]=\int d^{3} x\left\{\frac{1}{2} \Pi^{2}(\mathbf{x})+\frac{1}{2} \phi(\mathbf{x})\left(-\nabla^{2}+M_{\mathrm{cl}}^{2}\right) \phi(\mathbf{x})\right\}
$$

One obtains

$$
\begin{aligned}
C_{\mathrm{cl}}(t, \mathbf{k}, \mathbf{p})= & (2 \pi)^{3} \delta(\mathbf{p}+\mathbf{k}) \frac{T}{2 \omega_{\mathbf{p}}^{2}}\left\{\cos \left(\omega_{\mathbf{p}} t\right)\right. \\
& \left.-\frac{1}{2 \omega_{\mathbf{p}}^{2}}\left(\lambda T \int \frac{d^{3} k}{4 \omega_{\mathbf{k}}^{2}}-\delta M_{\mathrm{cl}}^{2}\right)\left(\omega_{\mathbf{p}} t \sin \left(\omega_{\mathbf{p}} t\right)+\cos \left(\omega_{\mathbf{p}} t\right)\right)\right\}
\end{aligned}
$$

The momentum integral is ultraviolet singular and is regularized by the cutoff $\mu$ :

$$
\int \frac{d^{3} k}{4 \omega_{\mathbf{k}}^{2}}=\frac{\lambda}{8 \pi^{2}} T \mu+\mathcal{O}\left(\frac{M_{\mathrm{cl}}^{2}}{\mu^{2}}\right)
$$

The contribution proportional to $t$ on the r.h.s. of eq. (2.14) is due to a resonance term in the r.h.s. of eq. (2.10) and has to vanish. This determines the mass counterterm (this self-consistent procedure is just equivalent to solving the Dyson equation with the polarization operator calculated by the tadpole graph in Fig. [1 in the classical limit):

$$
\delta M_{\mathrm{cl}}^{2}=\frac{\lambda}{4 \pi^{2}} T \mu+\mathcal{O}\left(\frac{M_{\mathrm{cl}}^{2}}{\mu^{2}}\right)
$$

Taking the Fourier transform of $C_{\mathrm{cl}}$ with respect to $t$ we obtain the classical Greens function in momentum space as

$$
G_{\mathrm{cl}}(p)=\frac{T}{\left|p^{0}\right|} 2 \pi \delta\left(p^{2}-M_{\mathrm{cl}}^{2}\right)
$$


This has to be compared with the quantum mechanical expression. The summation of the diagrams in Fig. 33 yields the real time propagator

$$
G(p)=\frac{i}{p^{2}-M^{2}+i \epsilon}+2 \pi \delta\left(p^{2}-M^{2}\right) \frac{1}{e^{\beta\left|p^{0}\right|}-1}
$$

with

$$
M^{2}=M_{0}^{2}+\frac{\lambda}{24} T^{2}
$$

The low energy limit eq. (2.18) is

$$
G(p) \sim \frac{T}{\left|p^{0}\right|} 2 \pi \delta\left(p^{2}-M^{2}\right)
$$

(It is also the classical limit. When substituting $p_{0} \rightarrow \hbar \omega, \mathbf{p} \rightarrow \hbar \mathbf{k}$, we see that the second term in Eq.(2.18) involves an extra power of $\hbar$ in the denominator compared to the first term when $\hbar \rightarrow 0$ ).

Ambjorn et al. have choosen the cutoff such that the energy densities of the quantum mechanical (eq. (1.1)) and the classical system (eq. (1.4)) are equal. With this choice, however, there is a mismatch of the self energies corresponding to the mass counterterms.

\section{A Formal Solution}

As was emphasized in the introduction, the quantities of interest which we wish to compute are real time correlation functions

$$
\begin{aligned}
C(t, \mathbf{x}) & =\langle\mathcal{O}(t, \mathbf{x}) \mathcal{O}(0)\rangle_{T} \\
& =\frac{\operatorname{Tr}\left\{e^{-\beta H} e^{i t H} \mathcal{O}(0, \mathbf{x}) e^{-i t H} \mathcal{O}(0)\right\}}{\operatorname{Tr} e^{-\beta H}} \\
& =\frac{\operatorname{Tr}\left\{e^{i t H} e^{-\beta H} \mathcal{O}(0, \mathbf{x}) e^{-i t H} \mathcal{O}(0)\right\}}{\operatorname{Tr} e^{-\beta H}}
\end{aligned}
$$

where $\mathcal{O}[\Phi(t, \mathbf{x})]$ is a local operator. This correlation function can be expressed as a path integral as

$$
C(t, \mathbf{x})=\int_{\left.\Phi\right|_{x^{0}=-i \beta}=\left.\Phi\right|_{x^{0}=0}}[d \Pi][d \Phi] \mathcal{O}(t, \mathbf{x}) \mathcal{O}(0) e^{i S}
$$

In this equation, the time variable $t$ is a real Minkowski time. The fields are periodic over the time $x^{0}=0$ to time $x^{0}=-i \beta$. The action has been written in terms of fields and their conjugate momenta in the form

$$
S=\int_{C} d x^{0} \int d^{3} x\left(\Pi(x) \partial_{0} \Phi(x)-\mathcal{H}(\Phi, \nabla \Phi)\right)
$$


where $\mathcal{H}$ is the Hamiltonian density. The contour $C$ of integration in time is shown in Figure 1 . It begins with a real time evolution from $x^{0}=0$ to the time $x^{0}=t$. It then takes a Euclidean path from $x^{0}=t$ to $x^{0}=t-i \beta$. Finally $x^{0}$ evolves backwards in time from $x^{0}=t-i \beta$ to $x^{0}=-i \beta$. The periodic boundary conditions for the trace are at the end points of the time contour.

This path integral representation yields the expression of Grigoriev and Rubakov in the stationary phase approximation $\delta \operatorname{Im}(i S)=0$. This requires $\Pi$ and $\Phi$ to be constant along the Euclidean interval of the contour, i.e. $\Pi\left(x^{0}, \mathbf{x}\right)=\Pi_{\text {in }}(\mathbf{x})$, $\Phi\left(x^{0}, \mathbf{x}\right)=\Phi^{\mathrm{in}}(\mathbf{x})$ and to be a solution of the classical equations of motion along the real time pieces. Then the phase vanishes in region $C_{I I}$, while the phases of regions $C_{I}$ and $C_{I I I}$ cancel each other. The exponential $\exp (i S)$ becomes $\exp \left\{-\beta H\left(\Pi^{\mathrm{in}}, \Phi^{\mathrm{in}}\right)\right\}$ and the only remaining integrations are those over $\Pi^{\text {in }}$ and $\Phi^{\text {in }}$, which are the initial values for the real time evolution in regions $C_{I}$ and $C_{I I I}$. Then the correlation function is

$$
C_{c l}(t, \mathbf{x})=\int\left[d \Pi^{\mathrm{in}}\right]\left[d \Phi^{\mathrm{in}}\right] e^{-\beta H\left(\Pi^{\mathrm{in}}, \Phi^{\mathrm{in}}\right)} \mathcal{O}_{c l}(t, \mathbf{x}) \mathcal{O}_{c l}(0)
$$

This treatment ignores quantum corrections completely. It can be justified if the only relevant modes are those with momenta much smaller than the temperature since then their occupation number is large and they can be treated classically. Since the calculation of Grigoriev and Rubakov is performed on a lattice, it involves a cutoff $\mu \sim 1 / a$, where $a$ is the lattice spacing. From the above argument $\mu$ should be much smaller than the temperature. Grigoriev and Rubakov have choosen $\mu \sim T$. If their result is $\mu$-independent, it is probably reliable. However, if it depends on $\mu$, it is sensitive to modes with momenta of order $T$, for which the classical treatment is not correct.

For the latter case we suggest the following procedure: One should choose the cutoff $\mu$ such that

$$
g T \ll \mu \ll T
$$

(This condition is written for gauge theories. For the $\lambda \phi^{4}$ theory the characteristic temperature-induced mass $g T$ should be substituted by $\lambda^{1 / 2} T$ ). The fields are decomposed into short wavelength modes and long wavelength modes:

$$
\Pi=\Pi_{S}+\Pi_{L}, \quad \Phi=\Phi_{S}+\Phi_{L}
$$

where $\Pi_{S}$ and $\Phi_{S}$ contain the Fourier componets with $|\mathbf{k}|>\mu$, and $\Pi_{L}, \Phi_{L}$ consist of those with $|\mathbf{k}|<\mu$. Then the $\Phi_{S}$ interact weakly among themselves and they can be treated as free fields in the background of $\Phi_{L}$, i.e. for these modes one can use the Gaussian approximation in the path integral. On the other hand, the condition $\mu \ll T$ ensures that the modes with $|\mathbf{k}|<\mu$ can be treated classically. 
Naively we could ignore the Gaussian fluctuations around a classical solution because it is higher order in coupling and, by assumption, the coupling is weak. In this case, however, there are corrections of order $g^{2} T^{2}$ to the classical equations of motion and these generate a huge correction for the small momenta we wish to consider. These terms arise from momenta which are much larger than the typical frequencies we wish to consider for our classical solution, and therefore the Gaussian fluctuations are dominated by the ultraviolet. The integration over these modes is equivalent to integrating out the high momentum modes and generating an effective theory of the low momentum modes.

Let us examine in more detail the reason why it is a good approximation to only do the Gaussian corrections to the path integral in the presence of the long wavelength modes. Consider modes with a typical momentum scale $\mu$. In this range of momentum, the occupation number of the modes, $T / \mu$, is large compared to 1 , but small compared to $1 / \mathrm{g}$. When these modes interact among themselves, the typical strength of interaction is of order $g T / \mu \ll 1$. Thus, nonlinear interactions among hard modes can be neglected, and the latter can be treated in the quadratic (Gaussian) approximation. In a diagrammatic language, it suffices to keep track only of the graphs involving 1-loop subgraphs with high internal momenta (the so called hard thermal loops).

We therefore seek to integrate out the short wavelength modes to obtain an effectively classical theory for the long wavelength modes. This gives

$$
C(t, \mathbf{x}) \simeq \int\left[d \Pi_{L}\right]\left[d \Phi_{L}\right] e^{i\left(S\left[\Pi_{L}, \Phi_{L}\right]+\delta S\left[\Pi_{L}, \Phi_{L} ; \mu\right]\right)} \mathcal{O}\left[\Phi_{L}(t, \mathbf{x})\right] \mathcal{O}\left[\Phi_{L}(0)\right]
$$

where

$$
\begin{aligned}
& e^{i \delta S\left[\Pi_{L}, \Phi_{L} ; \mu\right]} \\
& =\int\left[d \Pi_{S}\right]\left[d \Phi_{S}\right] \exp \left\{i \int d x ^ { 0 } \left(\int d^{3} x\left(\Pi_{S} \partial_{0} \Phi_{S}-\frac{1}{2} \Pi_{S}^{2}-\frac{\delta H\left(\Pi_{L}, \Phi_{L}\right)}{\delta \Phi(x)} \Phi_{S}(x)\right)\right.\right. \\
& \left.\left.\left.\quad-\frac{1}{2} \int d^{3} x \int d^{3} y \frac{\delta^{2} H\left(\Pi_{L}, \Phi_{L}\right)}{\delta \Phi\left(x^{0}, \mathbf{x}\right) \delta \Phi\left(x^{0}, \mathbf{y}\right)} \Phi_{S}\left(x^{0}, \mathbf{x}\right) \Phi_{S}\left(x^{0}, \mathbf{y}\right)\right)\right)\right\}
\end{aligned}
$$

The effective theory described by Eqs. (3.7, 3.8) will be the one for which the GR treament will be applied.

\section{Integrating Out the Hard Modes}

We have seen in the last section, that the effect of the hard modes is to generate an effective action for the long wavelength modes. Furthermore, it is sufficient to integrate them out in the Gaussian approximation. On the sight, this still appears as 
a tremendously difficult task. The exponent on the r.h.s of Eq. (3.8) contains terms linear in $\Phi_{S}$. They can be nonvanishing when $\Phi_{L}$ contains modes with momenta smaller but close to $\mu$ and the fields would have to be shifted, $\Phi_{S} \rightarrow \Phi_{S}+\delta \Phi_{S}$. Furthermore one has to sum all one loop diagrams with external momenta $\left|\mathbf{p}_{i}\right|<\mu$. The loop integration over $\mathbf{k}$ involves theta functions of the type $\Theta\left(\left|\mathbf{k}+\mathbf{P}_{i}\right|-\mu\right)$, where the $\mathbf{P}_{i}$ are linear combinations of the external momenta, for each internal line. However, these complications arise only for relatively hard modes in $\Phi_{L}$ with $\left|\mathbf{p}_{i}\right| \sim \mu$. In the previous section we have argued, that these modes are only weakly interacting and the corresponding contribution to $\delta S$ should be small. f

Therefore we can restrict ourselves to external momenta $\left|\mathbf{p}_{i}\right| \sim g T \ll \mu$. In this approximation $\delta S\left[\Pi_{L}, \Phi_{L} ; \mu\right]$ is given by the sum of the one loop diagrams with a factor $\Phi_{L}(x)$ for each external line, where the loop momentum is restricted by $|\mathbf{k}|>\mu$. Then it is possible, even for a nonabelian gauge theory, to extract the leading terms of all $n$-point functions. This is similar to the analysis by Braaten and Pisarski [18], who extracted the leading contribution proportional to $g^{2} T^{2}$. The only difference is that in our case there is a lower limit $\mu$ for the $|\mathbf{k}|$ integration, where $\mathbf{k}$ is the loop momentum.

For illustration, let us consider the simple case of the $\lambda \phi^{4}$-theory with the action

$$
S=\int_{C} d x^{0} \int d^{3} x\left\{\pi(x) \partial_{0} \phi(x)-\frac{1}{2}\left[\pi^{2}(x)+(\nabla \phi(x))^{2}+m^{2} \phi^{2}(x)\right]-\frac{\lambda}{4 !} \phi^{4}(x)\right\}
$$

Here the leading contribution in the effective action is given only by the diagram in Fig. 1. First the integration over $\pi(x)$ is performed. Then the free propagator for the short wavelength field is

$$
i D_{S}\left(x_{1}, x_{2}\right)=\int \frac{d^{4} k}{(2 \pi)^{4}} \Theta(|\mathbf{k}|-\mu) \rho_{0}(k) e^{-i k \cdot\left(x_{1}-x_{2}\right)}\left(\Theta_{C}\left(x_{1}^{0}-x_{2}^{0}\right)+n\left(k_{0}\right)\right)
$$

where $n\left(k_{0}\right)$ denotes the Bose distribution function, $\Theta_{C}\left(x_{1}^{0}-x_{2}^{0}\right)$ is the step function along the contour, and the spectral density $\rho_{0}(k)$ is given by

$$
\rho_{0}(k)=2 \pi \frac{1}{2 \omega_{k}}\left(\delta\left(k^{0}-\omega_{k}\right)-\delta\left(k^{0}+\omega_{k}\right)\right)
$$

with $\omega_{k}=\left(\mathbf{k}^{2}+m^{2}\right)^{1 / 2}$. We obtain

$$
\delta S=-i \frac{\lambda}{2} \int_{C} d x^{0} \int d^{3} x \phi^{2}(x) D_{S}(x, x)
$$

\footnotetext{
${ }^{5}$ In other words, the exact propagators and vertices with external momenta which are much larger than the characteristic temperature-induced mass coincide with tree propagators and vertices plus small corrections.
} 
The value of $D_{S}(x, x)$ is independent of $x^{0}$. In particular, it is the same on all three pieces of the contour. Therefore Eq. (4.4) can be written as

$$
\delta S=\int_{C} d x^{0} \int d^{3} x\left(-\frac{1}{2} \delta m^{2} \phi^{2}(x)\right)
$$

This expression contains an ultraviolet singularity, which is removed by a mass renormalization at zero temperature. Then, in the limit $m^{2} \ll \mu^{2}$, one obtains

$$
\delta m^{2}=\frac{\lambda}{24}\left(T^{2}-\frac{6}{\pi^{2}} \mu T\right)
$$

Taking into account Eq. (4.5) when calculating the correlation function has two effects. First, it modifies the equation of motion for the real time evolution in regions $C_{I}$ and $C_{I I I}$ of the time contour. Secondly, it changes the Hamiltonian on the Euclidean piece $C_{I I}$, which gives the thermal weight when taking the average over the initial conditions.

For $\lambda \phi^{4}$ theory, there are two equivalent ways to impose an ultraviolet cutoff in the classical problem. First, one can just expand the field in a Fourier series

$$
\phi(\mathbf{x}, t)=\sum_{\mathbf{n}} \phi_{\mathbf{n}}(t) e^{2 \pi i \mathbf{n} \mathbf{x} / L},
$$

write the classical equations of motion in terms of Fourier harmonics $\phi_{\mathbf{n}}(t)$ and impose the momentum cutoff $|\mathbf{k}|=2 \pi|\mathbf{n}| / L \leq \mu$. Technically, it is more convenient, however, to define the theory on a Hamiltonian lattice. As we show in Appendix B, the ultraviolet divergences in the one-loop lattice graph can be easily singled out, and the lattice analog of Eq.(1.11) is given by

$$
\begin{array}{r}
M_{\text {cl.lat. }}^{2}(T)=\frac{\lambda T a^{2}}{4} \int_{-\pi / a}^{\pi / a} \frac{d p_{1}}{2 \pi} \int_{-\pi / a}^{\pi / a} \frac{d p_{2}}{2 \pi} \int_{-\pi / a}^{\pi / a} \frac{d p_{3}}{2 \pi} \frac{1}{3-\sum_{i=1}^{3} \cos \left(a p_{i}\right)}= \\
\frac{\lambda T}{4(2 \pi)^{3} a} \int_{-\pi}^{\pi} d x \int_{-\pi}^{\pi} d y \int_{-\pi}^{\pi} d z \frac{1}{3-\cos x-\cos y-\cos z}=\frac{\lambda T}{4 a} \int_{0}^{\infty} d \alpha e^{-3 \alpha} I_{0}^{3}(\alpha)
\end{array}
$$

where $a$ is the 3-dimensional lattice spacing and $I_{0}(\alpha)$ is the exponentially rising variety of Bessel function. We see that the proper choice of $a$ is

$$
a=\frac{\pi^{2}}{\mu} \int_{0}^{\infty} d \alpha e^{-3 \alpha} I_{0}^{3}(\alpha)
$$

Then the formulae (4.8) and (1.11) match each other and the final result for the correlators (3.1) is cutoff-independent. 


\section{Gauge theories}

Let us discuss now whether it is possible, and if yes then how, to generalize the method worked out in the previous sections for $\lambda \phi^{4}$-theory to an $\mathrm{SU}(N)$ gauge theory. I As has already been mentioned in the Introduction, this is not so trivial since integrating out the modes with $|\mathbf{k}| \sim T$ does not simply result in a mass renormalization as in Eq.(1.10). One rather obtains nonlocal terms proportional to $g^{2} T^{2}$, which for the modes with $|\mathbf{k}| \leq g T$ are at least as large as the terms in the classical action (for the modes with $|\mathbf{k}| \sim g^{2} T$ they are even much larger!). Furthermore, there is a leading $g^{2} T^{2}$ contribution to all $n$-point functions. These terms have been identified in Ref. [18] and their generating functional is known as the hard thermal loop effective action. Since the resulting equations of motion are nonlocal, they are not appropriate for computing correlation functions a la Grigoriev and Rubakov.

Fortunately, this hard thermal loop effective field theory can be presented in a local form [19, 20]. In Ref. [19] it was formulated as a set of kinetic equations for the long wavelength gauge fields and the induced current $j$ of hard particles moving in the slowly varying background:

$$
\begin{aligned}
D_{\mu} F^{\mu \nu} & =j^{\nu} \\
j^{\nu}(x) & =2 g N \int \frac{d^{3} p}{(2 \pi)^{3}} v^{\nu} \delta n(\mathbf{p}, x)
\end{aligned}
$$

Here $D_{\mu}$ denotes the covariant derivative in the adjoint representation, $\delta n \equiv$ $\delta n^{a} t^{a}$ is the deviation of the thermal distribution of the hard particles from the equilibrium Bose distribution function $n_{0}(|\mathbf{p}|)$. Furthermore, $v^{\mu}$ is the velocity of massless particles with momentum $p, v^{\mu}=(1, \mathbf{v}), \mathbf{v}=\mathbf{p} /|\mathbf{p}|$. The kinetic equation for $\delta n$ is 1

$$
v^{\mu} D_{\mu} \delta n(x, \mathbf{p})=-g \mathbf{v} \cdot \mathbf{E} \frac{d n_{0}(|\mathbf{p}|)}{d|\mathbf{p}|}
$$

$\delta n$ depends nontrivially only on $\mathbf{v}$ and one can integrate eq. (5.3) over $|\mathbf{p}|$. On the

${ }^{6}$ We shall ignore for time being the Higgs fields and fermions. Possible effects due to their presence will be discussed at the end of the paper. Note that the studying of the correlator (1.5) makes sense also in hot $Q C D$ where it determines the rate of processes with chirality violation.

7 The equation system (5.1 [5.3) presents the nonabelian analog of the well-known Vlasov equations derived originally for the usual non-relativistic plasma [13. Silin generalized them for ultrarelativistic abelian plasma [24]. In Ref. 25] the kinetic equation approach has been used to study the properties of supersymmetric field theories at finite temperature. 
r.h.s. of equation (5.3) one has the integral

$$
\int_{0}^{\infty} d|\mathbf{p}||\mathbf{p}|^{2} \frac{d n_{0}(|\mathbf{p}|)}{d|\mathbf{p}|}=-\frac{1}{3} \pi^{2} T^{2}
$$

Then the induced current can be written in terms of

$$
w(x, \mathbf{v})=\frac{3}{g \pi^{2} T^{2}} \int \delta n(x, \mathbf{p})|\mathbf{p}|^{2} d|\mathbf{p}|
$$

as

$$
j^{\nu}(x)=2 m^{2}(T) \int \frac{d \mathbf{v}}{4 \pi} v^{\nu} w(x, \mathbf{v})
$$

where $m^{2}(T)=\frac{3}{2} \omega_{p l}^{2}(T)$, and $\omega_{p l}^{2}(T)=g^{2} T^{2} N / 9$ is the plasmon frequency. The function $w$ satisfies the equation

$$
v^{\mu} D_{\mu} w(x, \mathbf{v})=\mathbf{v} \cdot \mathbf{E}(x)
$$

By solving eq. (5.7) the induced current can be written as a functional of the gauge fields. Then the eq. (5.1) takes its nonlocal form obtained from the hard thermal loop effective action. The energy of the system of gauge fields and induced currents is given by

$$
H=\int d^{3} x \operatorname{Tr}\left\{\mathbf{E} \cdot \mathbf{E}+\mathbf{B} \cdot \mathbf{B}+2 m^{2}(T) \int \frac{d \mathbf{v}}{4 \pi} w(x, \mathbf{v}) w(x, \mathbf{v})\right\}
$$

An alternative local formulation has been given in Ref. [20] by introducing auxillary fields $G(x, \mathbf{v})$, which are $\mathrm{SU}(N)$ matrices satisfying the constraint $G(x,-\mathbf{v})=$ $G^{\dagger}(x, \mathbf{v})$. In the gauge $A_{0}^{a}=0$ with $D_{ \pm}=\partial_{0} \pm \mathbf{v} \cdot \mathbf{D}$, the Hamilton equations of motion are:

$$
\begin{aligned}
E_{i}^{a} & =\partial_{0} A_{i}^{a} \\
\partial_{0} E_{i}^{a}+\epsilon_{i j k}\left(D_{j} B_{k}\right)^{a} & =\int d \mathbf{v} v_{i}\left(J_{+}^{a}-J_{-}^{a}\right) \\
\left(D_{-} J_{+}\right)^{a} & =-\frac{m^{2}(T)}{8 \pi} E_{i}^{a} v_{i}
\end{aligned}
$$

where

$$
\begin{aligned}
J_{+}^{a} & =\frac{m^{2}(T)}{2 \pi} \operatorname{Tr}\left\{i t^{a}\left(D_{+} G\right) G^{-1}\right\} \\
J_{-}^{a} & =-\frac{m^{2}(T)}{2 \pi} \operatorname{Tr}\left\{i t^{a} G^{-1}\left(D_{-} G\right)\right\}
\end{aligned}
$$


The Gauss law constraint

$$
(\mathbf{D E})^{a}+\int d \mathbf{v}\left(J_{+}^{a}+J_{-}^{a}\right)=0
$$

should be imposed. The two local formulations of the hard thermal loop effective theory given by eqs. (5.1,5.6, 5.7) and eqs. (5.9 - 5.11) have been shown to be equivalent in Ref. 23.

An important remark is in order here. The one-loop graphs provide the dominant contribution to the effective Lagrangian when the external momenta are of order $g T$. When the external momenta become as small as $g^{2} T$ (and we presumably need that to solve the problem), the contribution of certain higher-order graphs to the effective vertices is of the same order as the contribution of hard thermal loops of Figs. 2, 7 [15].

Really, consider the two-loop graph for the gluon polarization operator in the kinematic region where the external momentum $q$ is of order $g^{2} T$ (see Fig. 5). The extra loop brings about the small factor $g^{2}$, but one can show that this loop involves the "resounding denominators": the propagators with momenta $p$ and $p+q$ bring about the large factor

$$
T^{4} \delta\left(p^{2}\right) \frac{1}{(p+q)^{2}} \sim \frac{T^{2}}{p q} \sim \frac{1}{g^{2}}
$$

which compensates the smallness $\sim g^{2}$ so that the contribution of the graph in Fig. 5 is unsuppressed. (An accurate analysis shows that such a large contribution appears only if taking into account hard thermal loops in the internal gluon propagator.)

In fact, all higher-loop ladder graphs contribute on the same footing. To find out the gluon polarization operator, the 3-point, 4-point etc. vertices at small momenta $\sim g^{2} T$, one has to resum all these ladders which is a formidable task (in Ref. [15], such a resummation has been done for the photon polarization operator in abelian theory).

Fortunately, however, we do not need to do that. The matter is that the integral for the graph in Fig. 5 is saturated by the region where the momentum of the rung of the ladder is soft: $|\mathbf{k}| \sim g T$ [15]. In our procedure, the integration should be done with the cutoff $|\mathbf{k}|>\mu \gg g T$ for all loop momenta. In that case, the contribution of the graph in Fig. 5 and all its higher-loop counterparts is suppressed again and can be safely ignored. Thus, we are left with the standard one-loop graphs in Figs. 2, 7 with loop momenta being restricted to be greater than the separation scale $\mu$.

Our suggestion is to solve numerically the equation system (5.9-5.11) rather than just equations of motion of the free Yang-Mills Hamiltonian. This would provide a proper account of the potentially dangerous high-momentum contributions. However, if one just uses the effective Hamiltonian (5.8) obtained after integration over all momenta in the hard thermal loops, the results would, generally, depend 
on the ultraviolet cutoff which has to be imposed. If one wants to construct a cutoff-independent algorithm, one should add to the Hamiltonian (5.8) $\mu$ - dependent counterterms so that the final result would not depend on $\mu$. However, to find out these counterterms explicitly is much more difficult task here than in the toy $\lambda \phi^{4}$ model.

For example, a simple-minded momentum ultraviolet cutoff is not suitable as it breaks gauge invariance. If trying to include the cutoff-dependent terms in the effective Hamiltonian (qualitatively, they modify the thermal mass $m^{2}(T) \sim g^{2} T^{2}$ by the terms $\sim g^{2} \mu T$ ), such a Hamiltonian would not commute with the Gauss law constraint and the classical problem just cannot be posed. See Appendix A for the detailed discussion.

Also, we do not know how to do it with a gauge-invariant lattice ultraviolet cutoff. First, it is not so trivial to construct a lattice version of the Nair system (5.9-5.11). But, even assuming it might be done, the major problem is that the lattice regularization breaks rotational invariance. As a result, the counterterms are also not rotationally invariant (see Appendix B for an illustrative calculation in scalar QED), their structure is complicated, and we are not able to write down their general form.

Thus, the only practical recipe we can suggest is to take the effective equations of motion for the full HTL Hamiltonian (5.8) and solve them numerically in the GR spirit with an ultraviolet cutoff $\mu \gg g T$. After averaging over initial conditions, we would get the correlator (1.5) . Though the result would generally depend on the cutoff $\mu$, we still hope that such a dependence would actually be weak. The arguments for this case and the corresponding discussion will be presented in the last section.

\section{Discussion}

But first let us discuss again the most important question: whether the procedure suggested here is really necessary, or perhaps the original procedure of Ref. [8] is still correct and the numerical calculations for the tree-level Hamiltonian (without integrating over high momentum modes) provide the results for the correlator (1.5) which do not really depend on ultraviolet cutoff.

At present, we do not know the answer to this question, and all we can do is to provide some arguments both pro and contra regarding the Ambjorn et al. case. Let us start with the pro arguments.

They come mainly from the observation that the physics associated with the baryon number non-conservation in electroweak theory at ultrahigh temperatures $T \gg T_{c}$ (and the physics of chirality non-conservation in hot QCD) is related to the momentum region $\sim g^{2} T$ and is essentially non-perturbative. The estimate (1.7) 
for the rate of such non-conservation depends only on the magnetic screening scale $\sim g^{2} T$ and not on the scale $g T$. It is conceivable then that all relevant momenta in the loops for the correlator (1.5) are of order $g^{2} T$. In that case, the results of the classical GR calculation are not sensitive to the ultraviolet region and just do not depend on the cutoff $\mu$.

Essentially the same can be argued in a slightly different way. We have seen that the dependence on $\mu$ generally comes together with the dependence on the plasmon mass scale $\sim g T$ [see Eqs. (4.6, A.19)]. But the latter is probably not relevant because the characteristic momentum scale, where the baryon number nonconservation occurs, is of order $g^{2} T$ which is much less than $m(T) \sim g T$. If so, the term $\sim \mu$ in $m^{2}(T, \mu)$ is also not relevant. And that means that the $\mu$-dependent pieces in the correlator (1.5) calculated in a classical procedure are also absent. If this guess is true, the results for the correlator (1.5) calculated with the tree Lagrangian and the results obtained in our procedure with the Braaten-Pisarski-Nair Lagrangian should just coincide in spite of that the theories look completely different. The huge effects due to effective mass and vertices renormalization should all cancel out in the end.8 In this case, all the effective HTL Lagrangian stuff may play the role of "(ultra-)violet hands on an enamel wall" [26] - beautiful but irrelevant for physics.

However, there is also an argument contra. It comes from the analysis of baryon nonconservation rate in the temperature region below the phase transition point. In this region, the quasiclassical approximation works and an analytical calculation is possible [6]. When $T_{c}-T \gg g T_{c}$,

$$
\Gamma(\Delta B \neq 0) \propto \exp \left\{-\frac{m_{W}(T)}{g^{2} T}\right\}
$$

where

$$
m_{W}^{2}(T)=m_{W}^{2}(0)\left(1-T^{2} / T_{c}^{2}\right)
$$

is the temperature-dependent $W$-mass. (At finite temperature, the notion of mass is not uniquely defined. The mass (6.2) is defined as a curvature of the effective

\footnotetext{
${ }^{8}$ There are two known examples where the cancellation of the effects related to hard thermal loops occurs in a physically observable quantity. One is the axial anomaly [27]. Individual graphs for the divergence of anomalous triangle are drastically changed if taking into account the renormalization of Green's function and/or vertices in the heat bath, but these effects completely cancel out in the sum of all graphs ( that just follows from the fact that the anomaly $\partial_{\mu} j_{\mu}^{5} \sim \operatorname{Tr}(G \tilde{G})$ is an operator identity which does not depend on whether one averages it over vacuum state or over a thermal ensemble). A similar cancellation of , at first sight, large effects due to so called anomalous damping occurs in the low-frequency electromagnetic polarization operator in quark-gluon plasma [15.
} 
potential, not as a pole of the propagator which has a completely different behavior [28] ).

Thus, the mass renormalization due to a high frequency thermal loop is essential when evaluating $\Gamma(\Delta B \neq 0)$ at $T \leq T_{c}$. Perhaps, these effects are also essential in the region $T \gg T_{c}$. In this case, the effective HTL Lagrangian is not a luxury but a neccessary tool and one should solve the equations of motion with the Hamiltonian (5.8) to determine the infrared asymptotics of the correlator (1.5).

Up to now we neglected the effects due to Higgs and fermion fields. The Higgs field is bosonic and can be treated in the same way as the gauge field: one has first to calculate hard thermal loops to determine the effective Lagrangian for the system including the gauge and the scalar fields and to write it down in a local form by introducing when necessary extra dynamic variables. One should then solve the equations of motion with this effective Lagrangian for soft modes.

In fact, Higgs fields have been taken into account in the numerical simulations in [8]. It was found that their inclusion practically does not affect the numerical results. That may be thought of as an argument supporting the conjecture that only magnetic gauge modes with momenta of order $g^{2} T$ are relevant for the problem.

Fermion fields play a different role because they cannot be treated classically. One has to include them however in the loops when deriving the effective Lagrangian for the gauge-Higgs system. If the naive GR calculation is ultraviolet-sensitive, also the terms in the Lagrangian due to fermion loops are important. If not - they are not.

Our suggestion to people who may wish to determine numerically the behaviour of the correlator (1.5) at very high temperatures (the calculations in [8] have been done in the region $T \sim T_{c}$ ) is the following: First, forget about the Higgs field and the fermion fields and do the calculations for a pure Yang-Mills system with the ultraviolet cutoff $\mu \gg g T$ (but it may be chosen much less than $T$ which is a considerable relief for a computer) and look whether the results depend on the cutoff. If they do not, the result is probably reliable. However, if they do, one has to redo the calculations with the Braaten-Pisarski-Nair effective Lagrangian in the way suggested in the paper. If the results obtained with this modified algorithm would not depend on the ultraviolet cutoff, one can be sure that they are the answer.

To find the baryon number nonconservation rate in the hot electoweak theory, one has first to derive the analog of Braaten-Pisarski Lagrangian with account of Higgs fields and fermions which is yet to be done.

Finally, let us discuss why one can hope that the modified procedure would lead to results which practically do not depend on the cutoff $\mu$. Essentially, it is due to the already mentioned fact that $\mu$-dependence in the GR procedure means the relevance of high momenta in the loops in which case it is natural to expect that the rate would depend not only on the scale $\sim g^{2} T$, but also on the plasmon mass scale $\sim g T$ which enters the HTL Hamiltonian (5.8). And that would mean that the estimate (1.7) is 
actually wrong (the heuristic arguments of [6] are physically appealing but they do not have the rank of a rigorous proof). In this case, however, one need not bother so much with carefullly subtracting the $\mu$-dependent counterterms because they affect results only slightly $\left(g^{2} \mu T \ll g^{2} T^{2}\right)$.

For sure, this argumentation is not rigourous and the possibility remains that the estimate (1.7) is still correct and the scale $\sim g T$ is irrelevant but the classical GR calculation is sensitive to the ultraviolet. In this case one cannot expect that the results obtained with the Hamiltonian (5.8) are $\mu$-independent, and the only way to solve the problem is to accurately isolate $\mu$-dependent counterterms in the effective Lagrangian with a gauge-independent (lattice) regularization procedure. We have seen that it is a very difficult task, and one should summon the forces to attack this problem only if more fortunate options - i) GR procedure is not sensitive to the ultraviolet and gives the correct answer and ii) Our modified procedure with the Hamiltonian (5.8) is not sensitive to the ultraviolet and gives the correct answer are ruled out.

\section{Acknowledgments}

We are indebted to J. Ambjorn, Yu.M. Makeenko, V. Rubakov, D. Schiff, M.S. Shaposhnikov, and N. Turok for illuminating discussions. A.S. acknowledges warm hospitality during his stay at the University of Minnesota when this work was started. support under DOE High Energy DE-AC02-83ER40105 and DOE Nuclear DE-FG02-87ER-40328.

\section{Appendix A. Soft gluon vertices with momentum}

\section{cutoff.}

This Appendix is devoted to the calculation of the hard thermal loops for multigluon vertices with an infrared cutoff for internal loop momentum $|\mathbf{p}| \geq \mu(g T \ll$ $\mu \ll T)$. Although, as we shall see, the effective Hamiltonian generated by these vertices breaks down gauge invariance and cannot be used to solve the problem, it is important to understand where the problem is. Also, this calculation may be interesting from a methodical viewpoint. We consistently use the real time formalism which is simpler and physically more transparent than the imaginary time formalism standardly used.

We start with estimating the contribution from the region $|\mathbf{p}| \geq \mu$ in the gluon polarization operator. 3 relevant graphs are depicted in Fig. 2. We work in the 
simplified Dolan-Jackiw (DJ) version [17] of the real time technique which is equivalent to the full-scale real time technique [11]-[14] in this particular problem. The DJ expressions for the gluon and the ghost propagators have the form (cf. Eq.(1.9)

$$
\begin{array}{r}
D_{\mu \nu}^{T, a b}(p)=-\delta^{a b}\left(g_{\mu \nu}-\xi \frac{p_{\mu} p_{\nu}}{p^{2}}\right)\left(\frac{i}{p^{2}+i 0}+2 \pi \delta\left(p^{2}\right) \frac{1}{e^{\beta\left|p_{0}\right|}-1}\right) \\
D_{\text {ghost }}^{T, a b}(p)=-\delta^{a b}\left(\frac{i}{p^{2}+i 0}+2 \pi \delta\left(p^{2}\right) \frac{1}{e^{\beta\left|p_{0}\right|}-1}\right)
\end{array}
$$

where $\xi$ is the gauge fixing parameter.

The 2-point vertex in the effective Lagrangian is given by the real part of the temperature-dependent contribution in the polarization operator which comes from the terms where the $\delta$-function insertion in the propagator is taken into account only once. In the limit when the external momentum $q$ is considered to be much smaller than the internal momentum $|\mathbf{p}| \sim \mu$, one can neglect $q$-dependence in the vertices and the expression is considerably simplified. Adding 3 graphs together, one gets after simple transformations

$$
\begin{array}{r}
\Pi_{\mu \nu}^{a b}(q)=2 g^{2} N \delta^{a b} \int \frac{d^{4} p}{(2 \pi)^{3}} \frac{2 p_{\mu} p_{\nu}-g_{\mu \nu} p^{2}}{(p+q)^{2}-p^{2}}\left[\delta\left[(p+q)^{2}\right] n_{B}\left(\left|p_{0}+q_{0}\right|\right)-\right. \\
\left.\delta\left(p^{2}\right) n_{B}\left(\left|p_{0}\right|\right)\right]+O(q)
\end{array}
$$

The imaginary time version of this formula can be found in [18]. Note that the result is $\xi$-independent. This is the notorious gauge-independence of hard thermal loops 18 .

Next, we expand in $q$ the denominator $(p+q)^{2}-p^{2}=2 p q+O\left(q^{2}\right)$ and the expression in the square brackets in the integrand in Eq. (A.2). In the latter, there are two terms coming from the expansion of $n_{B}\left(\left|p_{0}+q_{0}\right|\right)$ and of $\delta\left[(p+q)^{2}\right]$. When integrating over $d p_{0}$, we have to take into account two roots of $\delta$-function $p_{0}= \pm|\mathbf{p}|$ which give, however, identical contributions. Thus, it suffices to calculate the contribution of one of the roots $p_{0}=|\mathbf{p}|$.

The term involving $\delta^{\prime}\left(p^{2}\right)=\frac{1}{2 p_{0}} \frac{\partial}{\partial p_{0}} \delta\left(p^{2}\right)$ should be integrated by parts. The derivative $\frac{\partial}{\partial p_{0}}$ acts upon $n_{B}\left(p_{0}\right)$ and also on the structure

$$
S_{\mu \nu}(p)=\frac{2 p_{\mu} p_{\nu}-g_{\mu \nu} p^{2}}{p_{0}}
$$

It is not difficult to see that $\partial S_{\mu \nu}(p) / \partial p_{0}$ is nonzero only for the spatial components $\mu=i, \nu=j$. In the remaining integral over $d|\mathbf{p}|$ the infrared cutoff $|\mathbf{p}| \equiv \epsilon \geq \mu$ should be introduced. The loop integrals for $\Pi_{00}^{a b}, \Pi_{i 0}^{a b}, \Pi_{i j}^{a b}$ take the form

$$
\Pi_{00}^{a b}(q) \approx 2 g^{2} N \delta^{a b} \frac{1}{(2 \pi)^{3}} \int d \mathbf{v} \frac{\mathbf{q} \mathbf{v}}{q_{0}-\mathbf{q} \mathbf{v}} \int_{\mu}^{\infty} d \epsilon \epsilon^{2} \frac{d n_{B}(\epsilon)}{d \epsilon}
$$




$$
\begin{aligned}
\Pi_{0 i}^{a b}(q) \approx & 2 g^{2} N \delta^{a b} \frac{q^{0} q^{i}}{\mathbf{q}^{2}} \int d \mathbf{v} \frac{\mathbf{q} \mathbf{v}}{q_{0}-\mathbf{q} \mathbf{v}} \int_{\mu}^{\infty} d \epsilon \epsilon^{2} \frac{d n_{B}(\epsilon)}{d \epsilon} \\
\Pi_{i j}^{a b}(q) \approx & 2 g^{2} N \delta^{a b} \frac{1}{(2 \pi)^{3}} \int d \mathbf{v}\left\{v^{i} v^{j} \frac{\mathbf{q} \mathbf{v}}{q_{0}-\mathbf{q} \mathbf{v}} \int_{\mu}^{\infty} d \epsilon \epsilon^{2} \frac{d n_{B}(\epsilon)}{d \epsilon}\right. \\
& \left.-\frac{2}{3} \delta_{i j} \int_{\mu}^{\infty} d \epsilon \epsilon n_{B}(\epsilon)\right\}
\end{aligned}
$$

with $\mathbf{v}=\mathbf{p} / \epsilon$. We see that the integrals for $\Pi_{00}$ and $\Pi_{0 i}$ factorize in the product of two independent integrals: the energy integral and the angular integral. For $\Pi_{i j}$, the expression involves two different energy integrals and the factorization is not quite complete.

Without the infrared cutoff $\mu$ the two energy integrals would just coincide - one would be obtained from the other by integrating by parts. When $\mu \neq 0$, this is not the case:

$$
-\int_{\mu}^{\infty} d \epsilon \epsilon^{2} \frac{d n_{B}(\epsilon)}{d \epsilon}=\frac{\pi^{2}}{3}\left(T^{2}-\frac{3}{\pi^{2}} \mu T\right)
$$

and

$$
2 \int_{\mu}^{\infty} d \epsilon \epsilon n_{B}(\epsilon)=\frac{\pi^{2}}{3}\left(T^{2}-\frac{6}{\pi^{2}} \mu T\right)
$$

Since these integrals differ by a $\mu$-dependent term, the polarization tensor in Eq. (A.4) is not transverse (thereby the gauge invariance is lost). It is rather remarkable, however, that this non-transverse piece does not actually depend on the gauge parameter $\xi$ in the free thermal gluon propagators.

The appearance of non-transverse piece in the gluon polarization operator with momentum cutoff is not a surprise. Just recall that the usual zero-temperature photon polarization operator calculated with a momentum ultraviolet cutoff involves a quadratically divergent photon mass term.

What we are going to show next is that the troublesome mismatch (A.7, A.8) appears only for the 2-point function. For $n \geq 3$ all $n$-point functions retain the same form as without cutoff up to a universal renormalization of the coefficient. Consider first the 3-gluon vertex. In Feynman gauge there are two relevant diagrams depicted in Fig. 6 (The diagram containing a four gluon vertex does not contribute at leading order in q.). Let us calculate the real part of the effective vertex in the Dolan-Jackiw technique. Note that here not only the terms involving one thermal $\delta$-function but also the terms with the product of $3 \delta$-functions and 3 distribution functions contribute. The contribution of these $\delta \otimes \delta \otimes \delta$ term is huge $\sim g^{3} T^{3} / q_{\text {char }}^{2}$ where $q_{c h a r}$ is a characteristic external momentum. It is much greater than the tree vertex in the region $q_{c h a r} \sim g T$. Moreover, one can see that the integral for this contribution is singular in the infrared and is saturated by the internal momenta of order $q_{\text {char }}$. Thus, it is not a hard thermal loop at all! 
The resolution of this problem is known. The Dolan-Jackiw formalism gives the so called $\Gamma^{111}$ component of the vertex in the matrix real time formalism. What we need, however, is the retarded vertex $\Gamma^{R}$ (the one which is obtained by analytic continuation from the Euclidean region). $\Gamma^{R}$ presents the combination of $\Gamma^{111}$ and also other components. The term involving the product of 3 distribution functions cancels out in this combination [29]. One can show that the leading in $q$ terms in $\Gamma^{R}$ coincide with those in $\Gamma^{111}$ which involve only one temperature insertion If. As a result, the integral for the temperature contribution to the 3-point vertex has the following structure

$$
\begin{aligned}
\Gamma_{\mu \nu \lambda} \sim g^{3} \int & \frac{d^{4} p}{(2 \pi)^{3}} p_{1 \mu} p_{1 \nu} p_{1 \lambda}\left[\frac{\delta\left(p_{1}^{2}\right) n_{B}\left(\left|p_{10}\right|\right)}{\delta_{21} \delta_{31}}+\right. \\
& \left.\frac{\delta\left(p_{2}^{2}\right) n_{B}\left(\left|p_{20}\right|\right)}{\delta_{12} \delta_{32}}+\frac{\delta\left(p_{3}^{2}\right) n_{B}\left(\left|p_{30}\right|\right)}{\delta_{13} \delta_{23}}\right]
\end{aligned}
$$

where $\delta_{k l}=p_{k}^{2}-p_{l}^{2}$ and $p_{k}, k=1,2,3$ are the internal momenta in the loop. $\delta_{k l}$ have the order $\sim T q_{\text {char }} \sim g T^{2}$ and are small. We have also neglected the dependence on the external momenta in the tensor structure multiplying the square bracket in (A.9).

The individual terms in the r.h.s. of Eq. (A.9) have the order $\sim g^{3} T^{3} / q_{\text {char }}^{2}$ and are large. However, these large contributions cancel in the total sum due to the algebraic identity

$$
S_{3}(1,2,3)=\frac{1}{\delta_{21} \delta_{31}}+\frac{1}{\delta_{12} \delta_{32}}+\frac{1}{\delta_{13} \delta_{23}}=0
$$

As a result, the vertex has the normal HTL order $\sim g^{3} T^{2} / q_{\text {char }}$. To get this "nonleading" term, one has to expand the numerators of the fractions in Eq.(A.9) in the external momenta with respect to, say, the momentum $p_{1}$. As earlier, there will be the terms coming from the expansion of the distribution functions $n_{B}\left(\left|p_{20}\right|\right)$ and $n_{B}\left(\left|p_{30}\right|\right)$ which are proportional to external energies and also, potentially, the terms coming from the expansion of $\delta$-functions. The terms $\sim \delta^{\prime}\left(p_{1}^{2}\right)$ are dangerous as, when integrating by parts, the derivative may act upon the structure $\sim p_{\mu} p_{\nu} p_{\lambda} / p_{0}$ and provide the contribution involving the integral (A.8) rather than (A.7). That could give rise to some extra structures in the vertices and, as a result, in the effective equations of motion(A.18).

Fortunately, it does not happen. Really, the terms $\sim \delta^{\prime}\left(p^{2}\right)$ in $(\mathrm{A.9})$ are multiplied by

$$
-\frac{1}{\delta_{32}}-\frac{1}{\delta_{23}}=0
$$

\footnotetext{
${ }^{9}$ Now we understand what almost in the discussion preceding Eq.(1.9) meant.
} 
Thus, only the terms involving the expansion of $n_{B}\left(\left|p_{20}\right|\right), n_{B}\left(\left|p_{30}\right|\right)$ and thereby proportional to external energies and involving only the integral (A.7) appear.

Let us prove it in general (for a similar proof in imaginary time framework see [30]). The proof is inductive. Suppose we already know that, for the $n$-point vertex, the large individual contributions $\sim g^{n} T^{3} /\left(q_{c h a r}\right)^{n-1}$ coming from the graphs where $\delta$-function term is inserted in a particular internal line 1 cancel in the total sum, and terms coming from the expansion of the $\delta$-function also cancel out. Let us prove it for the $(n+1)$-point vertex.

The relevant graphs are shown in Fig. 7. The integral has the form

$$
\begin{array}{r}
\Gamma_{\mu_{1} \ldots \mu_{n+1}} \sim g^{n+1} \int \frac{d^{4} p}{(2 \pi)^{3}} p_{1 \mu_{1}} \ldots p_{1 \mu_{n+1}}\left[\frac{\delta\left(p_{1}^{2}\right) n_{B}\left(\left|p_{10}\right|\right)}{\delta_{21} \ldots \delta_{n+1,1}}+\right. \\
\left.\ldots+\frac{\delta\left(p_{n+1}^{2}\right) n_{B}\left(\left|p_{n+1,0}\right|\right)}{\delta_{1, n+1} \ldots \delta_{n, n+1}}\right]
\end{array}
$$

Let us prove that

$$
\begin{aligned}
S_{n+1}(1, \ldots, n+1)=\frac{1}{\delta_{21} \ldots \delta_{n+1,1}} & +\frac{1}{\delta_{12} \ldots \delta_{n+1,2}}+\cdots \\
& +\frac{1}{\delta_{1, n+1} \ldots \delta_{n, n+1}}=0
\end{aligned}
$$

To this end, present the second term in the r.h.s. of Eq.(A.13) as

$$
\frac{1}{\delta_{12} \ldots \delta_{n+1,2}}=\frac{1}{\delta_{12} \delta_{32} \ldots \delta_{n+1,1}}+\frac{1}{\delta_{1, n+1} \delta_{32} \ldots \delta_{n+1,2}}
$$

and do the same for the third, ..., and $n$-th term in the sum. Then it is not difficult to see that

$$
S_{n+1}(1, \ldots, n+1)=\frac{1}{\delta_{n+1,1}}\left[S_{n}(1, \ldots, n)-S_{n}(2, \ldots, n+1)\right]
$$

which is zero by the inductive assumption.

To get a nonzero result, one has to expand $\delta\left(p_{k}^{2}\right) n_{B}\left(\left|p_{k 0}\right|\right)$ in the integrand in (A.12) in the external momenta. The term proportional to $\delta^{\prime}\left(p_{1}^{2}\right)$ involves the factor

$$
-\frac{1}{\delta_{32} \ldots \delta_{n+1,2}}-\ldots-\frac{1}{\delta_{2, n+1} \ldots \delta_{n, n+1}} \equiv-S_{n}(2, \ldots, n+1)=0
$$

Thus, an additional non-transverse structure involving the difference of the integrals (A.8) and (A.7) appears only in the gluon polarization operator. Unfortunately, is it already bad enough to spoil the game.

\footnotetext{
${ }^{10}$ As earlier, only the terms with a single temperature insertion contribute in the retarded $(n+1)$ - point vertex.
} 
Let us try to write down the effective equations of motion corresponding to the $n$-point functions calculated above. By means of the time contour formalism of Sect. 3 one can see that indeed the retarded Greens functions enter the equations of motion ensuring causality. Thus we obtain

$$
\begin{gathered}
D_{\mu} F^{\mu 0}(x)=j^{0}(x) \\
D_{\mu} F^{\mu i}(x)=j^{i}(x)+\frac{1}{3 \pi^{2}} g^{2} N \mu T A^{i}(x)
\end{gathered}
$$

The current $j^{\mu}$ is given by eqs. (5.6), (5.7) with the plasmon mass $m_{p l}(T)$ replaced by $m_{p l}(T, \mu)$, where

$$
m_{p l}^{2}(T, \mu)=\frac{1}{6} g^{2} N\left(T^{2}-\frac{3}{\pi^{2}} \mu T\right)
$$

The extra term $\propto A^{i}$ in eq. (A.18) appears due to the mismatch (A.7)), (A.8) discussed above. Due to this term the equations of motion (A.18) are not consistent with Gauss' constraint (A.17). This means that choosing initial conditions satisfying the constraint (5.11), one cannot assure that this constraint will be fulfilled also at later times, and the classical problem cannot be consistently posed.

\section{Appendix B. Thermal loops on a Hamiltonian lat-}

\section{tice.}

We present here some illustrative calculations which display the way the cutoffdependence arises in the classical GR procedure with the lattice ultraviolet regularization. Unfortunately, the results of this study are negative - for the gauge theory in interest we were not able to extract explicitly the cutoff-dependent terms which is necessary to construct an explicit lattice-based cutoff-independent algorithm. But it is important to understand where the problem is. It is conceivable that after considerable future efforts such an algorithm would be eventually constructed.

Consider first the $\lambda \phi^{4}$ theory. The lattice Lagrangian 1 is

$$
\mathcal{L}_{\text {lat }}=\frac{a^{3}}{2} \sum_{\mathbf{n}} \dot{\phi}_{\mathbf{n}}^{2}-\frac{a}{2} \sum_{\mathbf{n} ; i=1,2,3}\left(\phi_{\mathbf{n}+\mathbf{e}_{i}}-\phi_{\mathbf{n}}\right)^{2}-\frac{\lambda}{4 !} a^{3} \sum_{\mathbf{n}} \phi_{\mathbf{n}}^{4}
$$

\footnotetext{
${ }^{11}$ It is more convenient to calculate graphs in the Lagrangian formulation, but one should remember that our lattice is 3 -dimensional and the time is real and continuous
} 
where $\mathbf{e}_{1}=(1,0,0)$ etc. To calculate graphs, we have to go over into the momentum representation (see [31] for details). Actually, the only thing we need to know here is the modified dispersive law

$$
p^{2} \equiv p_{0}^{2}-\frac{2}{a^{2}} \sum_{i=1}^{3}\left[1-\cos \left(a p_{i}\right)\right]=0
$$

The 3-momenta $p_{i}$ range within the limits

$$
-\pi / a \leq p_{i} \leq \pi / a
$$

Let us calculate the graph in Fig. 1 on the lattice in the real time formalism. The real time scalar lattice propagator has the form (cf. Eq.(1.9) )

$$
D_{T}(p)=\frac{i}{p^{2}+i 0}+2 \pi \delta\left(p^{2}\right) \frac{1}{e^{\beta\left|p_{0}\right|}-1}
$$

In the limit $a T \gg 1$, we can, as earlier, take the classical limit $T /\left|p_{0}\right|$ of the Bose distribution function after which the thermal contribution to the scalar mass acquires the form

$$
M_{\text {cl.lat. }}^{2}(T)=\frac{\lambda T a^{2}}{4} \int_{-\pi / a}^{\pi / a} \int_{-\pi / a}^{\pi / a} \int_{-\pi / a}^{\pi / a} \frac{d p_{1} d p_{2} d p_{3}}{(2 \pi)^{3}\left[3-\sum_{i=1}^{3} \cos \left(a p_{i}\right)\right]}
$$

With the help of the identities

$$
\frac{1}{A}=\int_{0}^{\infty} d \alpha e^{-\alpha A}, \quad \frac{1}{2 \pi} \int_{-\pi}^{\pi} d x e^{\alpha \cos x}=I_{0}(\alpha),
$$

the integral can be reduced to a one-dimensional form as spelled out in Eq. (4.8).

Let us go over now to the gauge theories. For illustrative purposes, we restrict ourselves with the case of scalar QED. The part of the lattice Lagrangian involving scalar fields is

$$
\begin{array}{r}
\mathcal{L}=a^{3} \sum_{\mathbf{n}}\left|\mathcal{D}_{0} \phi_{\mathbf{n}}\right|^{2}+a \sum_{\mathbf{n} ; i=1,2,3}\left(\phi_{\mathbf{n}+\mathbf{e}_{i}}^{*} U_{\mathbf{n}+\mathbf{e}_{i}, \mathbf{n}} \phi_{\mathbf{n}}+\right. \\
\left.\phi_{\mathbf{n}+\mathbf{e}_{i}} U_{\mathbf{n}+\mathbf{e}_{i}, \mathbf{n}}^{*} \phi_{\mathbf{n}}^{*}-\phi_{\mathbf{n}+\mathbf{e}_{i}}^{*} \phi_{\mathbf{n}+\mathbf{e}_{i}}-\phi_{\mathbf{n}}^{*} \phi_{\mathbf{n}}\right)
\end{array}
$$

where $\mathcal{D}_{0}=\partial_{0}-i e A_{0 \mathbf{n}}$ and $U_{\mathbf{n}+\mathbf{e}_{i}, \mathbf{n}}$ are complex fields living on the links of the lattice . (They have the meaning of parallel transporters: $U_{\mathbf{n}+\mathbf{e}_{i}, \mathbf{n}} \rightarrow \exp \left\{\right.$ iea $\left.A_{i}(\mathbf{x})\right\}$ in the continuum limit.)

The temperature contribution to the photon polarization operator is determined by the graphs in Fig. 8. In the continuum limit, it is given by the same integral as in Eq. A.2) . Let us impose the lattice ultraviolet cutoff and assume that $a T \gg 1 \gg a q$ where $q$ is the external momentum. 
Consider first the component $\Pi_{00}$ of the polarization operator. It is a little bit simpler than others because the vertices are the same as in the continuum theory. For $\Pi_{00}$ the only modification brought about by the lattice are the limited range of integration (B.3) over spatial momenta and the modified dispersive law (B.2). As a result, the integral for $\Pi_{00}$ reads

$$
\begin{array}{r}
\Pi_{00}=2 e^{2} T \int d p_{0} \int_{-\pi / a}^{\pi / a} \frac{d p_{1}}{2 \pi} \int_{-\pi / a}^{\pi / a} \frac{d p_{2}}{2 \pi} \int_{-\pi / a}^{\pi / a} \frac{d p_{3}}{2 \pi} \frac{2 p_{0}^{2}-p^{2}}{(p+q)^{2}-p^{2}} \\
{\left[\frac{\delta\left[(p+q)^{2}\right]}{\left|p_{0}+q_{0}\right|}-\frac{\delta\left(p^{2}\right)}{\left|p_{0}\right|}\right]}
\end{array}
$$

(where as earlier we neglected small $q$ compared to $p \sim 1 / a$ whenever possible). Expanding $1 /\left|p_{0}+q_{0}\right|$ and $\delta\left[(p+q)^{2}\right]$ in $q$, we get for the term $\propto 1 / a$ Ш

$$
\Pi_{00}=-\frac{2 e^{2} T}{a} \int_{-\pi}^{\pi} \int_{-\pi}^{\pi} \int_{-\pi}^{\pi} \frac{d^{3} s}{(2 \pi)^{3}} \frac{\sum_{i} q_{i} \sin s_{i}}{q_{0}-\frac{1}{\omega_{s}} \sum_{i} q_{i} \sin s_{i}} \frac{1}{\omega_{s}^{3}}
$$

where $s_{i}=a p_{i}$ and

$$
\omega_{s}^{2}=2 \sum_{i}\left(1-\cos s_{i}\right)
$$

And now we see that $\Pi_{00}$ is not the function of only $q_{0}$ and $\mathbf{q}^{2}=q_{1}^{2}+q_{2}^{2}+q_{3}^{2}$ as is the case in the continuum theory, but depends on each component of $\mathbf{q}$ separately. It is more or less obvious from the form of the integral. But if a reader cherishes a hope that it might not be true, he is welcome to expand the expression $(\overline{B .9})$ over $\mathbf{q} /\left|q_{0}\right|$ up to the fourth order and to be explicitly convinced that on top of the rotationally invariant structure $\sim\left(q_{1}^{2}+q_{2}^{2}+q_{3}^{2}\right)^{2}$ also the structure $\sim q_{1}^{2} q_{2}^{2}+q_{2}^{2} q_{3}^{2}+q_{1}^{2} q_{3}^{2}$ pops out with a non-zero coefficient.

For completeness, we present here also the results for the other components of $\Pi_{\mu \nu}:$

$$
\begin{aligned}
\Pi_{0 i} & =-\frac{2 e^{2} T}{a} q_{0} \int_{-\pi}^{\pi} \int_{-\pi}^{\pi} \int_{-\pi}^{\pi} \frac{d^{3} s}{(2 \pi)^{3}} \frac{\sin s_{i}}{q_{0}-\frac{1}{\omega_{s}} \sum_{i} q_{i} \sin s_{i}} \frac{1}{\omega_{s}^{3}} \\
\Pi_{i j} & =-\frac{2 e^{2} T}{a} q_{0} \int_{-\pi}^{\pi} \int_{-\pi}^{\pi} \int_{-\pi}^{\pi} \frac{d^{3} s}{(2 \pi)^{3}} \frac{\sin s_{i} \sin s_{j}}{q_{0}-\frac{1}{\omega_{s}} \sum_{k} q_{k} \sin s_{k}} \frac{1}{\omega_{s}^{4}}
\end{aligned}
$$

As the lattice regularization does not break gauge invariance, $\Pi_{\mu \nu}$ of Eqs. (B.9 B.11) is transverse: $q_{\mu} \Pi_{\mu \nu}=0$.

But, as the rotational invariance is broken, the cutoff-dependent counterterm in the 2-point vertex of the effective HTL Lagrangian is not presented as the sum of just two standard (transverse and longitudinal) tensor structures as was the case in the effective theory without cutoff. The same refers to counterterms for multiple-gluon vertices which we have not calculated.

12 cf. the expression (A.4) with the momentum cutoff. 


\section{References}

[1] N. Manton, Phys. Rev. D28 (1983) 2019, F. Klinkhammer, N. Manton, Phys. Rev. D30 (1984) 2212.

[2] G. t'Hooft, Phys. Rev. Lett. 37 (1976) 8.

[3] A. Linde, Phys. Lett. B70 (1977) 306.

[4] S. Dimopoulos, L. Susskind, Phys. Rev. D18 (1978) 4500

[5] V. Kuzmin, V. Rubakov, M. Shaposhnikov, Phys. Lett. B155 (1985) 36

[6] P. Arnold, L. McLerran, Phys. Rev. D36 (1987) 581.

[7] D.Yu. Grigoriev and V.A. Rubakov, Nucl.Phys. B299 (1988) 6719.

[8] J. Ambjorn et al, Nucl. Phys., B253 (1991) 346 ; J. Ambjorn and K. Farakos, Phys. Lett. B294 (1992) 248.

[9] J. Ambjorn, T. Askgaard, H. Porter and M. Shaposhnikov, Nucl. Phys. B353, 346 (1991); Phys. Lett. 244B, 479 (1990);J. Ambjorn, M. L. Laursen and M. Shaposhnikov, Nucl. Phys. B316, 483 (1989); Phys. Lett. 197B, 49 (1987).

[10] A. Bochkarev and P. De Forcrand, Phys. Rev. D47, 3476 (1993); Phys. Rev. D44, 519 (1991).

[11] P.M. Bakshi and K.T. Mahanthappa, J. Math. Phys. 4 (1963) 1, 12.

[12] L.V. Keldysh, Sov. Phys. - JETP 20 (1964) 1018.

[13] E.M. Lifshits and L.P. Pitaevsky, Physical Kinetics (Pergamon Press, Oxford, 1981).

[14] N.P. Landsman and Ch. G. van Wert, Phys. Repts 145 (1987) 141.

[15] V.V. Lebedev and A.V. Smilga, Physica A181 (1992) 187.

[16] A.V. Smilga, Physics of Atomic Nuclei 57 (1994) 550.

[17] L. Dolan and R. Jackiw, Phys. Rev. D9 (1974) 3320.

[18] E. Braaten and R. Pisarski, Nucl. Phys. B337 (1990) 569; Phys. Rev. D45 (1992) 1827; J. Frenkel and J.C. Taylor, Nucl. Phys. B334 (1990) 199; J.C. Taylor and S.M.H. Wong, Nucl. Phys. B346 (1990) 115.

[19] J. P. Blaizot, E. Iancu, SPhT/93-064 
[20] V.P.Nair, Preprint CCNY-HEP 2/94, hep-th/9403146.

[21] M. Alford, M. Gleiser, Phys. Rev. D48 (1993) 2838.

[22] Vacuum structure and QCD sum rules (M.A. Shifman, ed.) , North Holland, 1992.

[23] J. P. Blaizot, E. Iancu, Saclay-T94/041

[24] V.P. Silin, Sov. Phys. JETP 11 (1960) 1136.

[25] V.V. Lebedev and A.V. Smilga, Nucl. Phys. B318 (1989) 669.

[26] In "V. Bryusov, Sobranie sovhinenij v 7 tomah, Moscow, 1973 "(in Russian), v.1, p.35.

[27] H. Itoyama and A.H. Mueller, Nucl. Phys. B218 (1983) 349; A.V. Smilga, Phys. Rev. D45 (1992) 1378.

[28] A.V. Smilga, Phys. Lett, B222 (1989) 462.

[29] R. Baier, B. Pire, and D. Schiff, Z. Phys. C51 (1991) 581.

[30] R. Efraty, V. P. Nair, Phys. Rev. D74 (1992) 5601.

[31] M. Creutz, Quarks, gluons, and lattices. (Cambridge University Press, Cambridge, 1983). 


\section{Figure Captions}

Figure 1 The contribution to the propagator which yields the effective temperature dependent mass in $\lambda \phi^{4}$ theory.

Figure 2 The polarization contribution to the vector boson propagator at finite temperature. The dashed lines represent ghost propagators.

Figure 3 The sum of self energy insertions which yield the propagator in $\lambda \phi^{4}$ theory.

Figure 4 The contour of the time integral in the path integral representation for the correlation function.

Figure 5 Two-loop ladder graph contributing to the effective action for very soft momenta $q \sim g^{2} T$.

Figure 6 Leading contributions to the effective three gluon vertex in Feynman gauge. The dashed lines represent ghost propagators.

Figure 7 Hard thermal loop for a $(n+1)$ - gluon vertex. The vertical dashes stand for the temperature ( $\delta$-function) insertions. There are also similar graphs with internal ghost loop.

Figure 8 One-loop contributions to the photon polarization operator in scalar QED. 


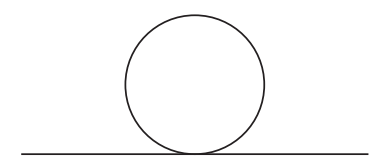

Figure 1
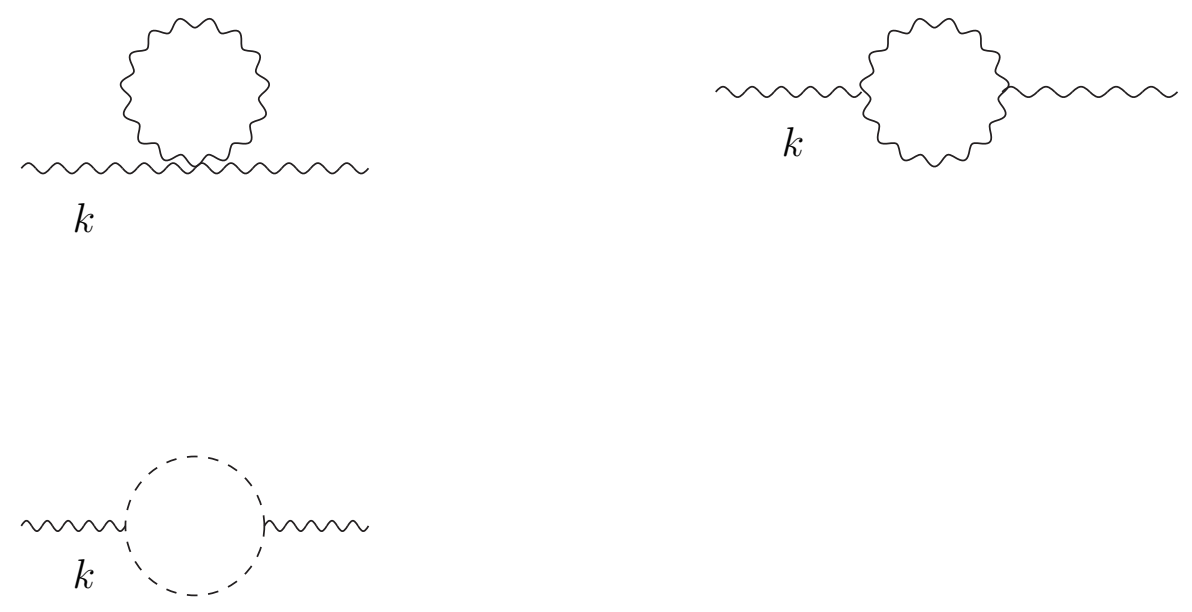

Figure 2 


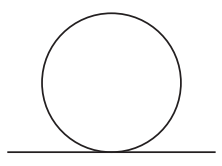

$+$

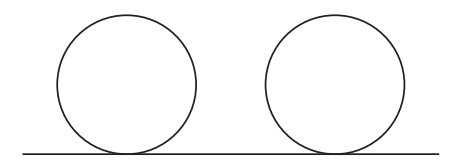

$+\quad \ldots$

Figure 3

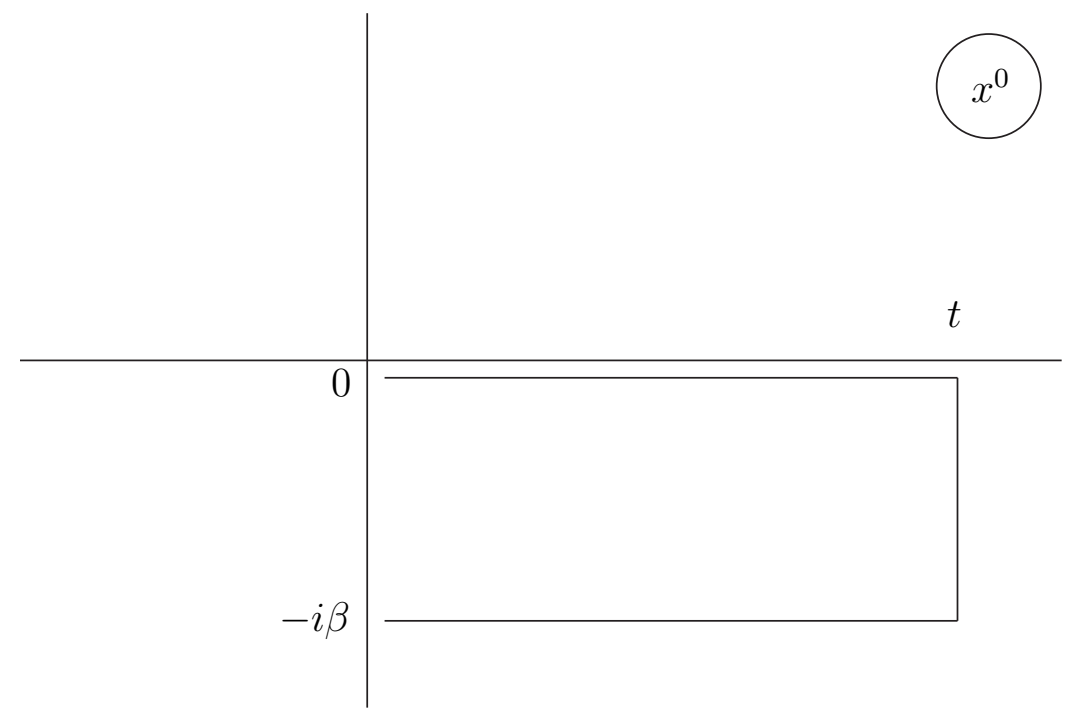

Figure 1 


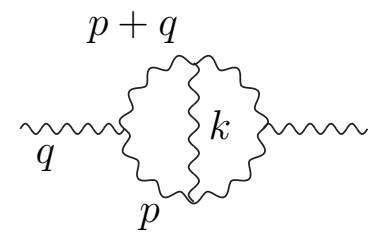

Figure 5
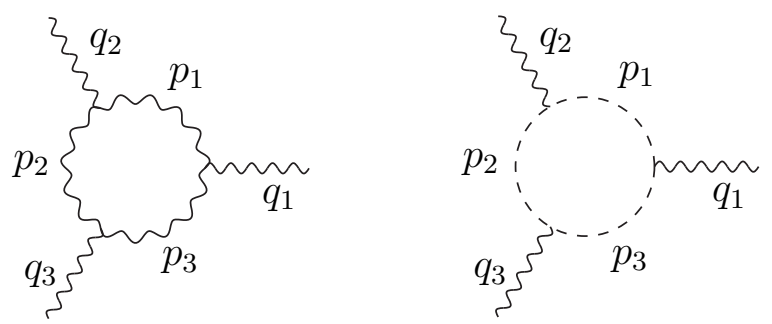

Figure 6 

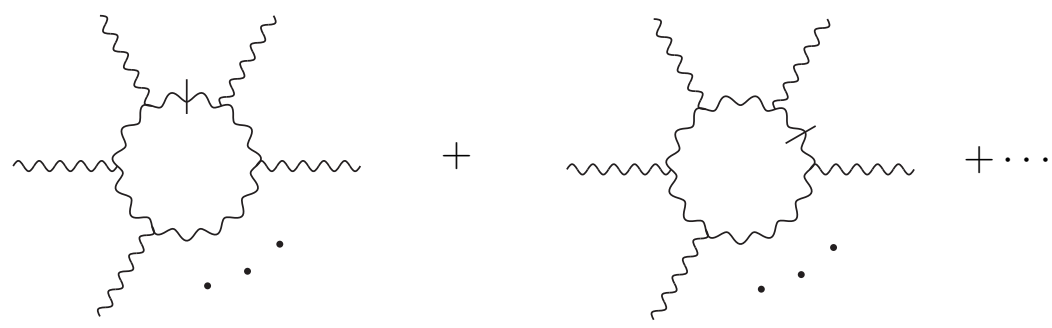

Figure 7
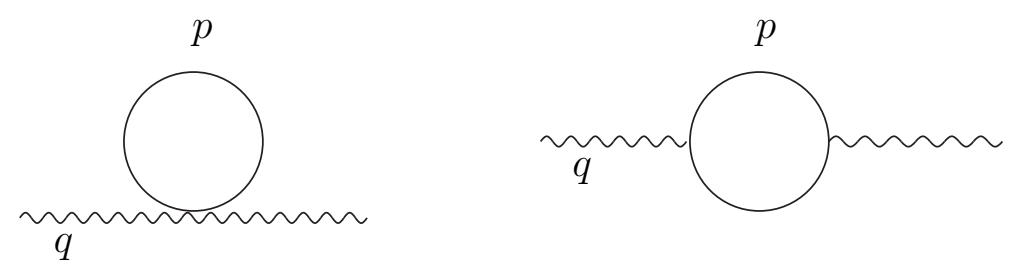

Figure 8 